\title{
The preventive and therapeutic application of garlic and other plant ingredients in the treatment of periodontal diseases (Review)
}

\author{
MASAHIRO OHTANI and TSUBASA NISHIMURA
}

\author{
Central Research Institute, Wakunaga Pharmaceutical Co., Ltd., Akitakata, Hiroshima 739-1195, Japan
}

Received August 1, 2019; Accepted September 3, 2019

DOI: $10.3892 /$ etm.2019.8382

\begin{abstract}
Since ancient times, pharmacologically active ingredients derived from natural sources, including plants and microbials have been used in the treatment of a wide array of diseases, such as atherosclerosis, diabetes mellitus and cancers. Herbal extracts and polyphenols are produced from herbs that contain a variety of ingredients, most of which exhibit anti-inflammatory, anti-oxidative and anti-microbial actions. Gingivitis is triggered by the infection of the periodontal tissues with periodontal disease-causing pathogens present in the dental biofilm. This is accompanied by weak inflammatory immune reactions in the gingiva. In periodontitis, prolonged and excessive inflammation results in the destruction of gingival connective tissue and in the resorption of alveolar bone, leading to tooth loss. There are a number of clinical reports showing the effectiveness of both herbal extracts and polyphenols on periodontal diseases when applied as a mouthwash or dentifrice into the oral cavity. However, to date, at least to the best of our knowledge, there is no clinical report available on the therapeutic effects of garlic or its extract on periodontal diseases, apart from a recent study, which reported that the intake of aged garlic extract (AGE) containing various pharmacologically active sulfur compounds, alleviated the symptoms of gingivitis clinically. The finding suggests that AGE may be a promising candidate for use in the treatment of periodontal diseases, although additional clinical trials are warranted to confirm this. In addition, further studies
\end{abstract}

Correspondence to: Dr Masahiro Ohtani, Central Research Institute, Wakunaga Pharmaceutical Co., Ltd., 1624 Shimokotachi, Koda-cho, Akitakata, Hiroshima 739-1195, Japan

E-mail: ootani_m@wakunaga.co.jp

Abbreviations: AGE, aged garlic extract; $\mathrm{CHX}$, chlorhexidine; ECG, epicatechin gallate; EGCG, epigallocatechin gallate; GI, gingival index; GBI, gingival bleeding index; IL-6, interleukin-6; IL-8, interleukin-8; LPS, lipopolysaccharide; NSAID, non-steroidal anti-inflammatory drug; $\mathrm{PGE}_{2}$, prostaglandin $\mathrm{E}_{2}$; ROS, reactive oxygen species; S1PC, $S$-1-propenylcysteine; SAC, $S$-allylcysteine; SAMC, $S$-allylmercaptocysteine; TNF- $\alpha$, tumor necrosis factor- $\alpha$

Key words: garlic, herb, inflammation, natural product, periodontal disease, polyphenol are required for the clarification of the basic molecular mechanisms through which AGE attenuates gingivitis. In this review, we summarize the beneficial effects of several natural compounds on periodontal disease and describe the possible applications of garlic ingredients in detail.

\section{Contents}

1. Periodontal disease

2. Evidence of the inhibitory effect of various naturally occurring products on gingivitis and periodontitis

3. Conclusions and future perspectives

\section{Periodontal disease}

Approximately $80 \%$ of adults worldwide suffer from gingivitis, which is the mild form of periodontal diseases (1). Gingivitis is initiated by infections caused by periodontal anaerobic pathogens, such as Porphyromonas gingivalis ( $P$. gingivalis), which are present in the oral biofilms, leading to prolonged inflammatory reactions in the gingival tissue (2). Without the mechanical removal of biofilm and/or supplemental treatment with antibiotics, the retraction of the gingiva occurs, followed by the resorption of the alveolar bone, and tooth loss (periodontitis) due to the chronic inflammation induced by inflammatory mediators, such as prostaglandin $\mathrm{E}_{2}\left(\mathrm{PGE}_{2}\right)(3,4)$. Furthermore, the accumulation of reactive oxygen species (ROS) produced by immune cells is the cause for the aggravation of gingival tissue injury (5).

Since ancient times, various natural products derived from plants have been utilized in the treatment of various disease symptoms (6). These products contain herbal extracts, polyphenols, garlic and etc. For the clinical treatment of periodontal disease, antimicrobials, such as chlorhexidine (CHX) have long been used (7). However, infections by bacteria resistant to antibiotics often become a severe clinical concern (5). Therefore, it is desirable to develop therapeutic substances for the treatment of periodontal diseases other than antibiotics. Promising candidates having no antibiotic resistance and few side-effects are naturally occurring products, such as polyphenols and garlic, which exert anti-inflammatory and antioxidant effects $(2-4,7)$. In this review, we summarize several natural products which have been shown to alleviate 
Table I. Effect of naturally occurring compounds in animal models of periodontitis and gingival cells.

\begin{tabular}{llc}
\hline Compounds & \multicolumn{1}{c}{ Pharmacological actions } & (Refs.) \\
\hline Herbal extracts (Kakkonto etc.) & Inhibition of $\mathrm{PGE}_{2}$ production in human gingival cells & $(4)$ \\
Sumac extract & Inhibition of alveolar bone resorption in rats & $(9)$ \\
Epicatechin gallate (ECG) & Inhibition of biofilm formation by P. gingivalis & $(11)$ \\
Epigallocatechin gallate (EGCG) & Inhibition of alveolar bone resorption in mice & $(12)$ \\
Theaflavin & Inhibition of alveolar bone resorption in rats & $(15)$ \\
& Inhibition of IL-6 and IL-8 secretion in human gingival cells & $(16,17)$ \\
Curcumin & Reduction of TNF- $\alpha$, IL-6, PGE levels in gingiva of rats & $(19,20)$ \\
Curcumin derivative (CMC 2.24) & Inhibition of alveolar bone resorption in rats & $(7)$ \\
\hline
\end{tabular}

Table II. Effect of garlic in gingival cells and gingivitis patients.

Compounds

Pharmacological actions

(Refs.)

Ethanol and aqueous garlic extract

Inhibition of growth of $P$. gingivalis and A. actonomycetescomitans

Garlic extract

Inhibition of growth of $P$. gingivalis

Allicin

Inhibition of growth of $A$. actonomycetescomitans and $F$. nucleatum

Diallyl sulfide

Aged garlic extract

Inhibition of growth of A. actonomycetescomitans

Alleviation of gingivitis in patients suffering from gingivitis

periodontal diseases mainly in clinical trials and in vivo experiments (Tables I and II). Furthermore, we introduce the anti-gingivitis effect of aged garlic extract (AGE) containing a variety of sulfur amino acids having the anti-inflammatory and antioxidant effects (2), and discuss the possibility for its application to treat periodontal diseases.

\section{Evidence of the inhibitory effect of various naturally oc- curring products on gingivitis and periodontitis}

Herbal extract. A number of herbal extracts consisting of plant-derived herbs have been used in the treatment of inflammatory diseases, such as stomatitis due to their wide range of biological activities, including anti-inflammatory effects, few side-effects and low costs (3-5). For the clinical treatment of periodontitis, non-steroidal anti-inflammatory drugs (NSAIDs) are systemically administered for the treatment of gingival inflammations $(4,8)$. However, NSAIDs often elicit side-effects, such as gastrointestinal dysfunction and bronchoconstriction (4). In human gingival cells, herbal extracts, such as kakkonto, shosaikoto and hangeshashinto have been shown to reduce the production of $\mathrm{PGE}_{2}$, which plays an important role in the progression of the gingival tissue degradation and alveolar bone loss, through the suppression of arachidonic cascade, similar to NSAIDs (4). Sumac (Rhus coriaria) is a spice widely used as an herbal medicine (5) and its extract has been shown to inhibit alveolar bone loss via its antioxidant properties in rats with periodontitis (9). Thus, herbal extracts may be possible candidates which can be used, in place of NSAIDs, to attenuate and prevent periodontal diseases.

Catechin. Green tea is known to contain 6 primary antioxidants, namely catechin, gallocatechin, epicatechin, epigallocatechin, epicatechin gallate (ECG) and epigallocatechin gallate (EGCG) (5). EGCG is a major polyphenol extracted from the leaves of Camellia sinensis (5) and is known to possess antioxidant and anti-bacterial activities (10). The $O$-methylated form of synthetic EGCG also exerts beneficial effects, such as the suppression of allergies (10). It has also been shown that ECG inhibits the biofilm formation of periodontal pathogens, such as $P$. gingivalis and Prevotella intermedia (11). It has been demonstrated that EGCG inhibits the resorption of alveolar bone induced by lipopolysaccharide (LPS) in mice suffering from periodontitis (12). In a preliminary clinical trial, the local application of green tea in the form of mouthwash or dentifrice was shown to produce an improved effect comparable to that of $\mathrm{CHX}$ on chronic periodontitis $(13,14)$.

Theaflavin. Theaflavin is a main polyphenol found in black tea made from the leaves of Camellia sinensis, and exerts anti-oxidant, anti-inflammatory and anti-tumor effects (15). In human gingival cells, theaflavin has been shown to decrease the secretion of interleukin (IL)- 6 and IL- 8 induced by LPS or tumor necrosis factor- $\alpha$ (TNF- $\alpha)(16,17)$. Recently, it was shown that theaflavin inhibited alveolar bone resorption in ligature-induced periodontitis rats, along with the reduction of immune cell infiltration and osteoclast formation in gingival tissues (15).

Curcumin. Turmeric is a yellow-orange spice derived from a rhizome of the plant Curcuma longa, and one of the main components in turmeric is curcumin (diferuloyl methane) (18). Clinical studies have demonstrated that curcumin exhibits a variety of therapeutic actions, such as anti-cancer, anti-platelet aggregation and vascular protective effects through its 
anti-inflammatory and anti-oxidant actions (18). Furthermore, curcumin has been shown to decrease blood cholesterol and triglyceride levels, despite its low bioavailability (18). In periodontitis, the oral administration of curcumin has been shown to reduce the levels of the inflammatory mediators, IL-6, TNF- $\alpha$ and PGE $_{2}$ in the gingival tissues of rats with both ligature- or LPS-induced periodontitis, whereas it was not shown to affect the resorption of alveolar bone $(19,20)$. On the other hand, chemically modified curcumin, but not curcumin per se, has been shown to inhibit bone loss in rats with LPS-induced periodontitis (7). In addition, gel and mouthwash containing the extract of Curcuma longa or curcumin have been found to decrease the several indices of gingivitis and the number of periodontal pathogens in clinical trials, along with or without the mechanical treatments, such as scaling and root planing (3).

Garlic components. Garlic has been used since ancient times to suppress the growth of bacteria, fungi and viruses (21). It has been demonstrated that garlic extract and its components attenuate atherosclerosis (22) and hypertension (23), and exert immunomodulatory and anti-tumor effects (24). It has also been found that the aqueous extract of garlic $(21,25)$ and its components, allicin (26) and diallyl sulfide (27), inhibit the growth of the periodontal pathogens, $P$. gingivalis, Aggregatibacter actinomycetemcomitans and Fusobacterium nucleatum in vitro. However, to date, at least to the best of our knowledge, no clinical study or in vivo experiment has been performed to determine whether garlic, the extracts of garlic and these garlic-derived substances can affect gingivitis or periodontitis. AGE, which is made by extracting and aging in water/ethanol solution for $>10$ months, contains a variety of pharmacologically active sulfur amino acids, such as $S$-allylcysteine (SAC), $S$-1-propenylcysteine (S1PC) and $S$-allylmercaptocysteine (SAMC) (28). AGE has been shown to attenuate atherosclerosis $(29,30)$ and lower hypertension $(31)$, possibly due to the anti-inflammatory $(28,32)$ and antioxidant effects $(33,34)$ of the sulfur compounds. Recently, the oral administration of AGE was shown to alleviate both the gingival index (GI) and gingival bleeding index (GBI) in a clinical trial (2), suggesting a promising application of AGE to relieve periodontal diseases. However, the mechanisms through which AGE attenuates gingivitis remain to be elucidated.

\section{Conclusions and future perspectives}

As described above, a variety of naturally occurring products, such as herbal extracts and polyphenols have been used in the treatment of periodontal diseases. A number of compounds are clinically applied directly into the oral cavity in the form of mouthwash or dentifrice. On the other hand, at least to the best of our knowledge, there is no report available to date describing the therapeutic efficacy of garlic or its extract, either clinically or in vivo, until a recent clinical trial, in which the intake of AGE was shown to significantly alleviate gingivitis (2). Thus, AGE may be a promising candidate for use in the treatment of periodontal disease. However, additional clinical trials are warranted to confirm this. Furthermore, further studies are required for the clarification of the basic molecular mechanisms through which AGE exerts its anti-gingivitis effects.

\section{Acknowledgements}

The authors are employees of Wakunaga Pharmaceutical Co. Ltd., which provided the funding for this study and would also like to thank Dr Takami Oka of Wakunaga Pharmaceutical Co. Ltd. for his helpful advice, encouragement, and critical reading of the manuscript.

\section{Funding}

This study was funded by Wakunaga Pharmaceutical Co. Ltd.

\section{Availability of data and materials}

Not applicable.

\section{Authors' contributions}

MO and TN conceived and designed this review article. MO drafted the manuscript. TN revised the manuscript. The authors have read and approved the final manuscript.

\section{Ethics approval and consent to participate}

Not applicable.

\section{Patient consent for publication}

Not applicable.

\section{Competing interests}

The authors declare that they have no competing interests.

\section{References}

1. Pulikkotil SJ and Nath S: Effects of curcumin on crevicular levels of IL-1 $\beta$ and CCL28 in experimental gingivitis. Aust Dent J 60: 317-327, 2015.

2. Zini A, Mann J, Mazor S and Vered Y: The efficacy of aged garlic extract on gingivitis-A randomized clinical trial. J Clin Dent 29: 52-56, 2018.

3. Safiaghdam H, Oveissi V, Bahramsoltani R, Farzaei MH and Rahimi R: Medicinal plants for gingivitis: A review of clinical trials. Iran J Basic Med Sci 21: 978-991, 2018.

4. Ara T, Nakatani S, Kobata K, Sogawa N and Sogawa C: The biological efficacy of natural products against acute and chronic inflammatory diseases in the oral region. Medicines (Basel) 5: 122,2018

5. Ramesh A, Varghese SS, Doraiswamy JN and Malaiappan S: Herbs as an antioxidant arsenal for periodontal diseases. J Intercult Ethnopharmacol 5: 92-96, 2016.

6. Benso B, Rosalen PL, Alencar SM and Murata RM: Malva sylvestris inhibits inflammatory response in oral human cells. An in vitro infection model. PLoS One 10: e0140331, 2015.

7. Wang HH, Lee HM, Raja V, Hou W, Iacono VJ, Scaduto J, Johnson F, Golub LM and Gu Y: Enhanced efficacy of chemically modified curcumin in experimental periodontitis: Systemic implications. J Exp Pharmacol 11: 1-14, 2019.

8. Salvi GE and Lang NP: Host response modulation in the management of periodontal diseases. J Clin Periodontol 32 (Suppl 6): 108-129, 2005.

9. Sağlam M, Köseoğlu S, Hatipoğlu M, Esen HH and Köksal E: Effect of sumac extract on serum oxidative status, RANKL/OPG system and alveolar bone loss in experimental periodontitis in rats. J Appl Oral Sci 23: 33-41, 2015. 
10. Tominari T, Matsumoto C, Watanabe K, Hirata M, Grundler FM Miyaura $\mathrm{C}$ and Inada $\mathrm{M}$ : Epigallocatechin gallate (EGCG) suppresses lipopolysaccharide-induced inflammatory bone resorption, and protects against alveolar bone loss in mice. FEBS Open Bio 5: 522-527, 2015.

11. Asahi Y, Noiri Y, Miura J, Maezono H, Yamaguchi M, Yamamoto R, Azakami H, Hayashi M and Ebisu S: Effects of the tea catechin epigallocatechin gallate on Porphyromonas gingivalis biofilms. J Appl Microbiol 116: 1164-1171, 2014.

12. Tominari $T$, Ichimaru $R$, Yoshinouchi $S$, Matsumoto $C$ Watanabe K, Hirata M, Grundler FMW, Inada M and Miyaura C: Effects of $O$-methylated (-)-epigallocatechin gallate (EGCG) on LPS-induced osteoclastogenesis, bone resorption, and alveolar bone loss in mice. FEBS Open Bio 7: 1972-1981, 2017.

13. Hrishi T, Kundapur P, Naha A, Thomas B, Kamath S and Bhat G: Effect of adjunctive use of green tea dentifrice in periodontitis patients - A randomized controlled pilot study. Int J Dent Hyg 14: 178-183, 2016.

14. Hirasawa M, Takada K, Makimura M and Otake S: Improvement of periodontal status by green tea catechin using a local delivery system: A clinical pilot study. J Periodontal Res 37: 433-438, 2002

15. Wu YH, Kuraji R, Taya Y, Ito H and Numabe Y: Effects of theaflavins on tissue inflammation and bone resorption on experimental periodontitis in rats. J Periodontal Res 53: 1009-1019, 2018.

16. Lombardo Bedran TB, Morin MP, Palomari Spolidorio D and Grenier D: Black tea extract and its theaflavin derivatives inhibit the growth of periodontopathogens and modulate interleukin- 8 and beta-defensin secretion in oral epithelial cells. PLoS One 10: e0143158, 2015.

17. Hosokawa Y, Hosokawa I, Ozaki K, Nakanishi T, Nakae H and Matsuo T: Tea polyphenols inhibit IL-6 production in tumor necrosis factor superfamily 14-stimulated human gingival fibroblasts. Mol Nutr Food Res 54 (Suppl 2): S151-S158, 2010.

18. Nagpal M and Sood S: Role of curcumin in systemic and oral health: An overview. J Nat Sci Biol Med 4: 3-7, 2013.

19. Guimarães MR, Coimbra LS, de Aquino SG, Spolidorio LC, Kirkwood KL and Rossa C Jr: Potent anti-inflammatory effects of systemically administered curcumin modulate periodontal disease in vivo. J Periodontal Res 46: 269-279, 2011.

20. Guimarães MR, de Aquino SG, Coimbra LS, Spolidorio LC, Kirkwood KL and Rossa C Jr: Curcumin modulates the immune response associated with LPS-induced periodontal disease in rats. Innate Immun 18: 155-163, 2012.

21. Shetty S, Thomas B, Shetty V, Bhandary R and Shetty RM: An in-vitro evaluation of the efficacy of garlic extract as an antimicrobial agent on periodontal pathogens: A microbiological study. Ayu 34: 445-451, 2013.

22. Tsui PF, Lin CS, Ho LJ and Lai JH: Spices and atherosclerosis. Nutrients 10: 1724, 2018

23. Ried K: Garlic lowers blood pressure in hypertensive individuals, regulates serum cholesterol, and stimulates immunity. An updated meta-analysis and review. J Nutr 146: 389S-396S, 2016.
24. Moutia M, Habti N and Badou A: In vitro and in vivo immunomodulator activities of Allium sativum L. Evid Based Complement Alternat Med 2018: 4984659, 2018

25. Bakri IM and Douglas CW: Inhibitory effect of garlic extract on oral bacteria. Arch Oral Biol 50: 645-651, 2005.

26. Bachrach G, Jamil A, Naor R, Tal G, Ludmer Z and Steinberg D: Garlic allicin as a potential agent for controlling oral pathogens. J Med Food 14: 1338-1343, 2011.

27. Velliyagounder K, Ganeshnarayan K, Velusamy SK and Fine DH: In vitro efficacy of diallyl sulfides against the periodontopathogen Aggregatibacter actinomycetemcomitans. Antimicrob Agents Chemother 56: 2397-2407, 2012.

28. Suzuki JI, Kodera Y, Miki S, Ushijima M, Takashima M, Matsutomo $\mathrm{T}$ and Morihara N: Anti-inflammatory action of cysteine derivative S-1-propenylcysteine by inducing MyD88 degradation. Sci Rep 8: 14148, 2018.

29. Matsumoto S, Nakanishi R, Li D, Alani A, Rezaeian P, Prabhu S, Abraham J, Fahmy MA, Dailing C, Flores F, et al: Aged garlic extract reduces low attenuation plaque in coronary arteries of patients with metabolic syndrome in a prospective randomized double-blind study. J Nutr 146: 427S-432S, 2016.

30. Zeb I, Ahmadi N, Nasir K, Kadakia J, Larijani VN, Flores F, Li D and Budoff MJ: Aged garlic extract and coenzyme Q10 have favorable effect on inflammatory markers and coronary atherosclerosis progression: A randomized clinical trial. J Cardiovasc Dis Res 3: 185-190, 2012.

31. Ried K, Travica N and Sali A: The effect of aged garlic extract on blood pressure and other cardiovascular risk factors in uncontrolled hypertensives: The AGE at Heart trial. Integr Blood Press Control 9: 9-21, 2016.

32. Anandasadagopan SK, Sundaramoorthy C, Pandurangan AK, Nagarajan V, Srinivasan K and Ganapasam S: S-Allyl cysteine alleviates inflammation by modulating the expression of NF- $\kappa \mathrm{B}$ during chromium (VI)-induced hepatotoxicity in rats. Hum Exp Toxicol 36: 1186-1200, 2017.

33. Hiramatsu K, Tsuneyoshi T, Ogawa T and Morihara N: Aged garlic extract enhances heme oxygenase-1 and glutamate-cysteine ligase modifier subunit expression via the nuclear factor erythroid 2-related factor 2-antioxidant response element signaling pathway in human endothelial cells. Nutr Res 36: 143-149, 2016.

34. Tsuneyoshi T,Kunimura K and Morihara N: S-1-Propenylcysteine augments BACH1 degradation and heme oxygenase 1 expression in a nitric oxide-dependent manner in endothelial cells. Nitric Oxide 84: 22-29, 2019.

This work is licensed under a Creative Commons Attribution-NonCommercial-NoDerivatives 4.0 International (CC BY-NC-ND 4.0) License. 